\title{
THE POISSON'S RATIO OF THE NUCLEUS PULPOSUS IS STRAIN DEPENDENT
}

\author{
Mark D Farrell, Philip E Riches \\ Bioengineering Unit, University of Strathclyde \\ United Kingdom
}

\section{Introduction}

The nucleus pulposus (NP) is the hydrated central region of the intervertebral disc (IVD) and is composed of a network of collagen fibrils, elastic fibres, proteoglycans and interstitial fluid. Its function is to transfer load to the surrounding annulus fibrosus (AF) during compression. In mechanical studies, the NP is generally modelled as homogenous and isotropic to reduce computational complexity. However, the validity of these assumptions has not been fully validated. Biphasic or poroelastic theory has been widely used in the modelling of hydrated soft tissue and, as such, many models describe the NP as an incompressible fluid and adopt a Poisson's ratio value of $\sim 0.5$, whilst others have adopted values between 0.35 and 0.49 (Meakin and Hukins, 2001). Only one study has attempted to directly determine the Poisson's ratio of NP, via an optical measurement technique, and reported a significantly higher value of $0.62 \pm 0.15$ (Cloyd et al., 2007) which may have been due to sample swelling. Therefore, the objective of this study was to characterise the true Poisson's ratio of the nucleus pulposus with strain.

\section{Methods}

Eight cylindrical plugs of NP tissue $(\varnothing 5.5 \mathrm{~mm}$, height $1544 \pm 252 \mu \mathrm{m}$ ) orientated in the axial direction were harvested from bovine tail discs. The plugs were subjected to unconfined compression, between polished glass platens lubricated with an aqueous lubricant mixed with $3 \mathrm{M} \mathrm{NaCl}$, to $20 \%$ axial strain in $5 \%$ increments, at a $30 \mu \mathrm{m} / \mathrm{s} \mathrm{ramp}$ speed with 1 hour hold periods. Lateral displacement and radial strain was determined using biaxial videoextensometry (Bose Electroforce 3200 with 2D video extensometer). The apparent Poisson's ratio $\left(v_{\mathrm{A}}\right)$ was calculated by using radial strain results obtained immediately after the ramp phase, whilst true Poisson's ratio $\left(v_{\mathrm{T}}\right)$ was calculated by using radial strain results obtained at the end of the hold phase. $v_{\mathrm{A}}$ represents the Poisson's ratio of the tissue, including both solid and fluid, whilst $v_{\mathrm{T}}$ is the Poisson's ratio of the solid phase in isolation.

To validate the equipment and methodology, a silicone analogue (Rhodorsil RTV 3428, UK) was subjected to the same test. Further, tissue-platen and analogue-platen static friction coefficients were determined using a tilting technique.

\section{Results}

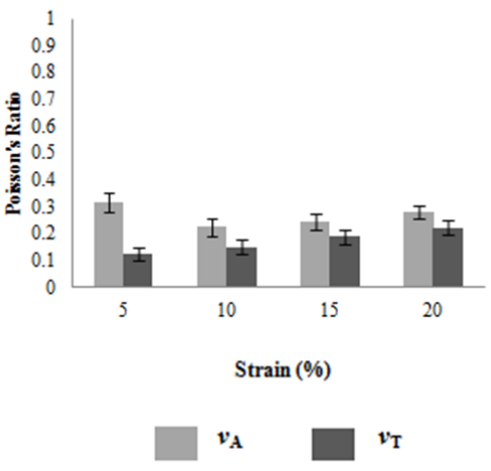

Figure 1: Poisson's ratio results

With increasing strain, $v_{\mathrm{T}}$ increased and lateral recoil $\left(v_{\mathrm{A}}-v_{\mathrm{T}}\right)$ decreased. True Poisson's ratio varied between 0.12 and 0.22 . The silicone analogue had $v=0.49$. Static friction coefficients for NP tissue and the analogue on the glass platen were $0.44 \pm 0.08$ and $0.26 \pm 0.05$ respectively.

\section{Discussion}

The result for the silicone analogue, together with tissue recoil, suggests a valid experimental setup with minimal friction effects. During the ramp phase, fluid pressure gradients drive fluid radially with the viscous drag on the solid matrix imposing radial strain over and above Poisson's ratio effects. In the hold phase, the radial stress in the collagen and elastic fibres, as a result of the flow induced viscous drag, facilitates tissue recoil during the hold phase. However, with increasing strain, tissue densification and reduction in pore volume may explain the increase in $v_{\mathrm{T}}$.

\section{References}

Cloyd et al., 2007. Material properties in unconfined compression of human nucleus pulposus, injectable hyaluronic acid-based hydrogels and tissue engineering scaffolds. Eur Spine J, 16, 1892-8.

Meakin et al., 2001. Replacing the nucleus pulposus of the intervertebral disk: prediction of suitable properties of a replacement material using finite element analysis. Journal of Materials Science-Materials in Medicine, 12, 207-213. 